Original Research

\title{
Are professional pharmacy services being offered for free in pharmacies? A feasibility study exploring the use of a time motion study in New Zealand
}

\author{
Yasmin H. ABDUL AZIZ (iD), \\ Susan J. HEYDON iD, Stephen B. DUFFULL (iD), Carlo A. MARRA (iD). \\ Published online: 31-Jul-202
}

Received (first version): 2-May-2021

Accepted: 11-Jul-202

\begin{abstract}
Background: Pharmacists report to be providing patient-focused clinical services for which they receive no remuneration. Limited literature exists about unfunded services leading to difficulties in ascertaining an appropriate study design for such research.

Objective: This study aims to assess the appropriateness of a proposed study design before launching a nationwide study to investigate the provision of unfunded patient care services.

Methods: A multi-methods approach was utilised consisting of (1) continuous time motion study in community pharmacies (2) semi structured patient interviews (3) patient follow up (4) semi structured interviews with pharmacy owners/managers. All observations of unfunded patient care services were recorded, numerically coded and descriptively analysed. Semi structured interviews were audio recorded and transcribed verbatim. A semantic thematic analysis was carried out. Appropriateness of study design was dictated by the ability to characterise services and obtain patient perceptions.

Results: Ten pharmacies took part in the feasibility study, across the city of Dunedin, New Zealand, representing a range of different practice settings and demographics. Ten patients were interviewed and six responded to follow up. Both pharmacy and patient recruitment proved challenging due to concerns around disruption to workflow and patient privacy. A continuous observation time motion study was found to be appropriate as it minimises disruption to workflow with no additional work required from the pharmacy teams.

Conclusions: A continuous observation time motion study proved to be an appropriate method to investigate the provision of unfunded services on a national scale. The findings of the study suggest design changes such as length of observation time, increasing patient recruitment and additional patient questions to enhance the nationwide study.
\end{abstract}

\section{Keywords}

Community Pharmacy Services; Pharmaceutical Services; Patient Care; Remuneration; Professional Practice; Workflow; Pharmacies; Pharmacists; Research Design; Feasibility Studies; Qualitative Research; New Zealand

\section{INTRODUCTION}

There is a global push from funders and policy makers for pharmacists to move away from the traditional dispensing, supply and distribution roles to a role of patient-centred care. $^{1-3}$ One of the fundamental barriers to the provision of patient centred care has been identified as the lack of remuneration attached to these services. ${ }^{4-9}$

In recent years and on a global level, increased media coverage reports that pharmacists are providing many patient care services that are deemed 'unfunded'. ${ }^{10-13} \mathrm{We}$ define these services as those provided through pharmacies for which no remuneration from the government, insurance companies or payments from patients is received. ${ }^{14}$ There has been little description of the nature of these patient focused services with limited literature exploring this area. An earlier scoping study ascertained a model for unfunded pharmacy service

Yasmin H. ABDUL AZIZ. BPharm, PGCertPharm, PhD. School of Pharmacy, University of Otago. Dunedin (New Zealand). yasmin_abdulaziz@outlook.com

Susan J. HEYDON. BA(Hons), MA, PhD, DHMSA. Senior Lecturer. School of Pharmacy, University of Otago. Dunedin (New Zealand).

susan.heydon@otago.ac.nz

Stephen B. DUFFULL. DipPharm(CIT) MPharm, PhD, FNZCP

FISOP, MPS, RegPharmNZ. Professor. School of Pharmacy,

University of Otago. Dunedin (New Zealand).

stephen.duffull@otago.ac.nz

Carlo A. MARRA. BSc(Pharm) PharmD, PhD. Professor and Dean.

School of Pharmacy, University of Otago. Dunedin (New Zealand). carlo.marra@otago.ac.nz provision in New Zealand. ${ }^{14}$ Based on narratives provided by pharmacists, a number of unfunded services provided by community pharmacies in New Zealand were identified. These services were found to belong in three categories (1) Standalone unfunded services, pertaining to services which are not recognised or remunerated by the government or insurance companies (2) Services funded elsewhere pertaining to services funded in one geographical region but not another and finally (3) Leakages from the funding model, pertaining to fully funded services provided in an unfunded setting such as services with capitation. These services were mostly described as screening and monitoring, the management of common and minor ailments, and wound care. ${ }^{14}$ The study highlighted the fragmented provision of unfunded services throughout New Zealand based on national and local funding models. Furthermore, the findings questioned equity in healthcare access across the country. This study relied on pharmacists' self-reporting, where biases stemming from over-reporting and providing socially desirable ideas may have occurred. Furthermore, little is known about the details of these unfunded services including the frequency of provision, the type of staff providing such services and the labour costs associated with these services.

Literature is lacking in the area of real-time studies examining unfunded pharmacy service delivery in community pharmacies. Studies examining the real-time delivery of unfunded services are crucial to understand the 
value and the impact on patient health outcomes that these unfunded services achieve.

Numerous methods exist enabling the real time investigation of service delivery, from continuous observation time motion studies to work sampling involving the observation of set tasks (e.g., medication preparation). ${ }^{15-17}$ In order to investigate unfunded services across New Zealand, it is essential to ascertain an appropriate method for data collection. This would ensure that the study design would allow for investigating the costs and patient health outcomes associated with unfunded pharmacy services. A feasibility study was designed to investigate the appropriateness of a time motion study in the evaluation of the provision of unfunded services in community pharmacies in New Zealand.

Health research studies often involve large numbers of subjects and locations rendering them resource intensive. Feasibility studies are useful in health research in order to evaluate proposed study designs to ensure effective use of resources. ${ }^{18}$ They are designed to assess the effectiveness of data collection, analysis and establish whether the study design meets the aims of the intended study. ${ }^{19}$ Feasibility studies are generally conducted with a smaller number of participants and provide inference on study design and power, such as the number of sites, subjects and follow up processes before undertaking the larger study. Feasibility studies also help identify additional variables of interest that should therefore be considered in the larger scale study. ${ }^{18}$ It should be noted that feasibility studies only inform the study design - they are not intended to evaluate the outcome of interest - rather this is left to the main study. ${ }^{18,20}$

The aim of this feasibility study was to ascertain an appropriate study design for a larger scale nationwide study about unfunded pharmacy services. This study assessed the feasibility of utilising a continuous observation time motion study to investigate the provision of unfunded services. Additionally it aimed to ascertain (1) an appropriate and effective method for the recruitment of pharmacies and patients (2) the required length of observation time to make conclusions about practice and (3) whether qualitative interviews with pharmacy owners and managers would yield any additional information about financial sustainability of these services.

\section{METHODS}

\section{Study design}

Qualitative and quantitative methods were utilised, this consisted of four components (1) Continuous observation time motion study, (2) Patient interviews, (3) Patient follow up and, (4) Pharmacy owner/manager interviews.

The continuous observation time motion study investigated unfunded service provision in real-time and characterised these services. Onsite patient interviews explored patient perceptions and the value they place on unfunded services provided in the pharmacy. Patient follow up was conducted to ascertain whether the service patients received from the pharmacy helped resolve their clinical issue. Finally, pharmacy owners/managers were also interviewed to investigate the methods utilised to achieve financial viability.

Eligible participants were community pharmacies and patients in metropolitan Dunedin, New Zealand.

Ethical approval was obtained from the University of Otago Human Ethics Committee, Dunedin, New Zealand (reference number H18/097).

The appropriateness of the study design was assessed based on (1) recruitment rate, (2) the ability to capture the critical information (e.g., documenting the types of unfunded services offered and the proportion of time expended providing these services) and (3) the need to carry out all elements in the study (i.e., could one or more of the 4 study elements be dropped without loss of study performance e.g., pharmacy owner/manager interviews).

\section{Participant recruitment}

A list of community pharmacies was obtained from the official Dunedin city phone book. Pharmacies were contacted by the primary author through telephone and invited to take part in the time motion study. The study would involve the primary author observing the provision of unfunded services provided in the respective pharmacies across two business days.

Pharmacies were selected based on the demographics catered to (defined by the age and socioeconomic status of the population in the area), the type of business model utilised by the pharmacy (e.g., retail, dispensary or highvolume discounted sales) in order to be representative of the demographic of pharmacies in New Zealand. Dunedin (population: 126,255) was selected due to convenience as well as being nationally representative of different pharmacy groups/types and patient demographics. Recruitment of pharmacies ceased when data saturation occurred which was when no new information about the services were being identified.

Potential patient participants were identified as those receiving an unfunded service on the day of the continuous observation study. Patients were approached and asked if they would like to take part in a short interview to share their views about the service they had received. Pharmacy owners/managers of participating pharmacies were interviewed as part of the feasibility study. Interviews with patients and pharmacy owners/managers were semistructured in nature allowing for the exploration of broad topics and perceptions around access to unfunded services and maintaining financial viability (Table 1 ).

\section{Data collection}

All data collection was carried out by the primary investigator.

Time motion study

Observations were recorded on a paper case report form with time data recorded from a stopwatch. 'Observations' pertain to an unfunded clinically-focused patient interaction or service provided by any member of the pharmacy staff. The case report form accounts for information about: the type of service provided; the type of 


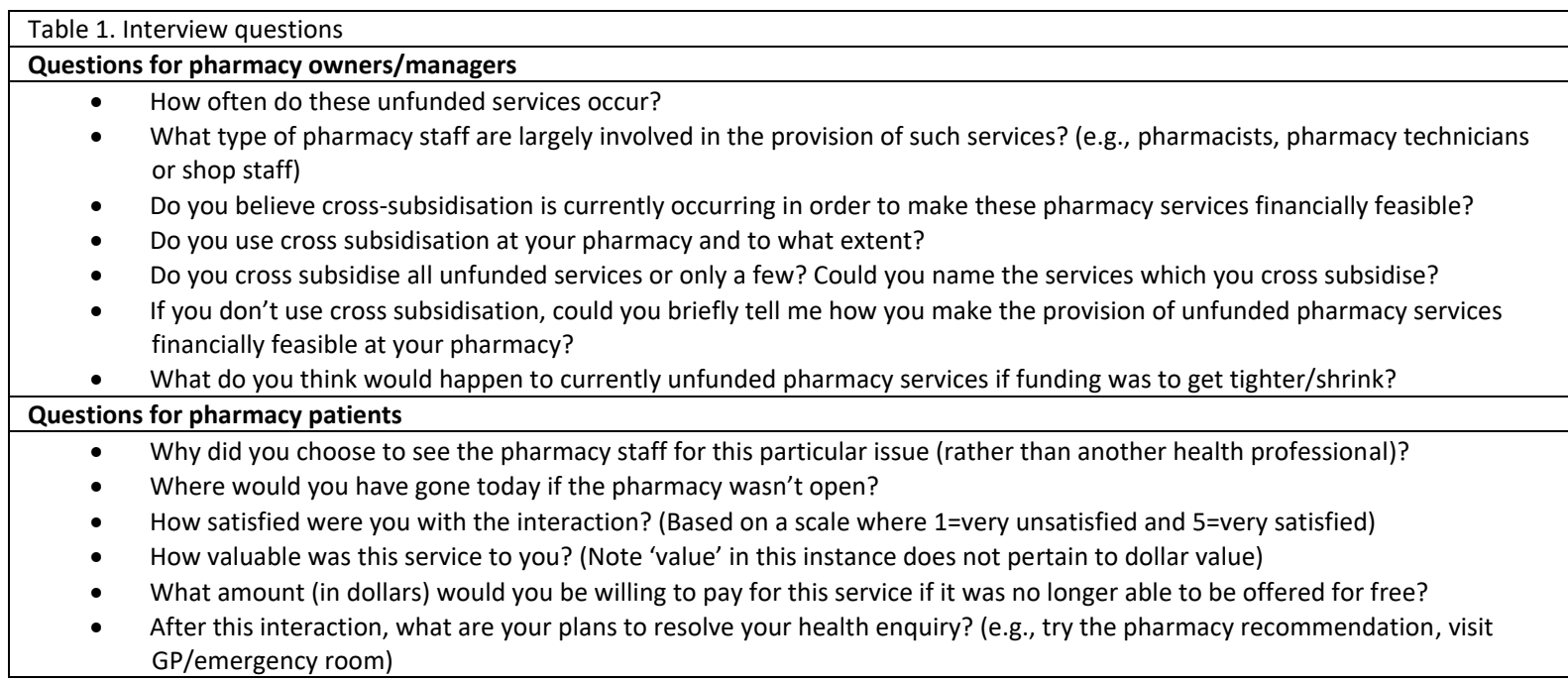

staff member providing the service; the time taken to provide the service and whether a fee was paid for receiving the service. Observations were carried out over eight business hours per day. The time motion study was conducted over weekdays and weekends to ensure the representation of the different patient profiles during the course of a week.

\section{Patient interviews}

Interviews were conducted with a number of patients immediately after the patient had accessed the unfunded service. The interviews were designed to be five minutes in duration, owing to the busy nature of the pharmacy and the short times spent by patients in the pharmacy. The interviews were allowed to conclude naturally when no new patient perceptions were shared. All interviews were audio recorded.

\section{Patient follow up}

Patient follow up occurred by contacting the patient on the phone number provided. Follow up occurred seven days after observing the patient receive the unfunded services from the pharmacy. Patients were contacted up to three times in the period of one week; if no response was achieved after the third phone call, the patient would be deemed lost to follow up. Voice messages were sent to the patient after the initial non-response call but only if there was an identifiable voicemail greeting. No confidential information was disclosed in voice or text messages.

\section{Pharmacy owner/manager interviews}

These interviews were conducted during a quiet period in the pharmacy to avoid losing observations of unfunded services. The interviews were designed to be of 30 minutes duration, but were allowed to conclude naturally when no new information about financial viability was emerging. All interviews were audio recorded.

\section{Coding and data analysis}

All interview data were transcribed verbatim by the primary author. One third of the total transcriptions and codes were validated by a co-author to ensure accuracy.

Qualitative data were coded using the inductive coding method in the QSR International NVIVO software (version
11). Consensus on the codes assigned was reached through review and discussion with a co-authors. The coded data allowed for identifying key ideas and perceptions held by patients around the access of unfunded services from pharmacies as well the patients' perceived value of these services. Similarly, coded data were used to identify pharmacy owner/manager views on achieving and maintaining the financial viability of the provision of unfunded services. Data were then analysed using semantic thematic analysis.

Observational data collected were numerically coded and entered into the IBM SPSS software (version 25). Each observation was coded based on the presenting condition, service provided, outcome of query, and the associated time for service delivery based on the type of staff providing the service (i.e., pharmacist, pharmacy technician, intern etc.). The following equation was used to ascertain the proportion of an average working day spent providing unfunded services.

$$
\text { Proportion of time taken }=\frac{\text { Total time providing unfunded services }(\text { hours })}{\text { Total staff time }(\text { hours })} \times 100
$$

'Staff' refers to those from one category i.e., pharmacists, pharmacy technicians etc. Total staff time refers to the total number of working hours for a particular staff type employed at the pharmacy in question.

\section{RESULTS}

The continuous observation time motion study yielded a total of 148 observations of unfunded pharmacy service provision across the 112 hours (14 business days) of observation. Data collection occurred from October 2018 until March 2019. Data saturation from individual pharmacies was also reached by the end of the first business day. As a result of this finding the duration of the time motion study could be reduced to one day per pharmacy whilst increasing the sample size to capture a larger variety of pharmacies catering to differing populations and therefore differing health needs.

Rarely was more than one unfunded service provided by different staff members and could not be simultaneously recorded due to only one investigator being present. 


\begin{tabular}{|c|c|}
\hline Pharmacy characteristics ( $n=7)$ & $\mathbf{N}$ \\
\hline \multicolumn{2}{|l|}{ Size of pharmacy } \\
\hline Large pharmacy & 3 \\
\hline Medium pharmacy & 2 \\
\hline Small pharmacy & 2 \\
\hline \multicolumn{2}{|l|}{ Location within Dunedin } \\
\hline Suburb & 2 \\
\hline Central Business District (CBD) & 3 \\
\hline Mall & 1 \\
\hline Medical Centre & 1 \\
\hline \multicolumn{2}{|l|}{ Type of pharmacy } \\
\hline Chain pharmacy & 2 \\
\hline Independent pharmacy & 4 \\
\hline Supermarket & 1 \\
\hline \multicolumn{2}{|l|}{ Income area** } \\
\hline Low socioeconomic & 2 \\
\hline Middle socioeconomic & 1 \\
\hline High socioeconomic & 2 \\
\hline Mixed socioeconomic & 2 \\
\hline
\end{tabular}

Ten pharmacies were invited to take part in the feasibility study, seven $(70 \%)$ of which agreed to participate. Participating pharmacies were geographically distributed across Dunedin. Pharmacies represented a range of different practice settings and demographics served as highlighted in Table 2.

Pharmacy staff had initial concerns around patient privacy and possible disruption of workflow. Whilst some pharmacies were quick to agree to take part, others cited short staffing to be the main reason for declining the invitation to participate as they perceived staff involvement in data collection. This perception led to a firmer emphasis both orally and in the written information sheet that no additional tasks were to be undertaken by the staff and the intent was for minimal disruption to workflow. The method utilised in this study was found to be amenable by the participating pharmacy staff, as it did not require them to undertake additional tasks during their working hours.

A total of thirteen patients were invited to take part in the on-site interviews of which ten patients $(77 \%)$ consented. Interview durations ranged from one minute to five minutes in duration, based on patients' responses.

Of the ten patients interviewed six patients $(60 \%)$ responded to the follow up telephone conversation. Follow ups were approximately five minutes in duration. Responses were recorded verbatim on a paper notepad.

All six pharmacy owners/managers agreed to be interviewed, two of whom were dispensary managers and the remaining interviewees were pharmacy owners. Although there were seven participating pharmacies, one interviewee owned two pharmacies in different geographical locations in Dunedin, serving different demographics of patients.

All pharmacy managers/owners represented a range of different age groups and years of ownership and management experience. Interview durations were between five to eleven minutes.

Interviews yielded no new information as compared the earlier scoping study about methods to achieve financial viability other than cross subsidisation achieved through 'up-selling' over the counter products. ${ }^{14}$

\section{DISCUSSION}

The feasibility of a continuous observation time motion study, qualitative interviews and patient follow up were assessed in preparation for a nationwide study to investigate the provision of unfunded services across New Zealand. This is the first study to investigate the provision of unfunded services in real-time. It should be noted that this study was informed and guided by the findings of the earlier scoping study. ${ }^{14}$ The utilisation of qualitative and quantitative methods allowed for the thematic analysis of patient perceptions of these services and the quantification of the provision of these services in practice. The results showed that implementing a continuous observation time motion study was effective in gathering data about such services. The continuous observation method, when applied, provided a reflection of the unfunded services provided in practice as unlike the work sampling technique it not only captured pre-determined services but allowed for the investigation of other services that may have not been previously captured in earlier pharmacies in the feasibility study. Methodological findings were in line with those in the literature that demonstrate the effectiveness of time motion studies in pharmacy research. ${ }^{17,21,22}$

The use of a continuous observation time motion study addressed one of the challenges encountered in the recruitment of pharmacies namely concerns around staff undertaking additional tasks. The feasibility study further ruled out relying on pharmacist self-reporting as a method of data collection based upon the feedback gathered in the recruitment phase of the study. This method can be cumbersome and introduce undesirable additional paperwork for the staff which was found to be a major concern to pharmacy staff prior to consenting to this feasibility study. Furthermore by not implementing this method it eliminated self-reporting bias and avoided bias resulting from reporting socially desired responses. ${ }^{23}$ In line with the challenges found in recruiting pharmacies, it became clear during this study that staff did not want to be involved in carrying out additional tasks during or after their interactions with patients. This finding was appropriate as we did not want the methods to alter workflow within the pharmacy, but capture what an average working day consists of. Additionally, pharmacy owners and managers had concerns about staff members being asked to undertake additional tasks for the purpose of this study. From this experience it was decided that recruitment on a national level would utilise a 'facilitator' pharmacist who, through word of mouth, would advertise the study to pharmacists in the area to help overcome challenges in recruitment experienced during the feasibility study. Special attention would be given to ensure that the pharmacies recruited represented a range of demographics as well as practice settings.

Three components of the study namely, time motion observation study, patient interviews and follow ups were found to strengthen the study when conducted together, as a more holistic view of unfunded services were achieved. Data yielded from each component would allow for further 
characterisation of service provision on a national level. Two business days were initially arbitrarily selected to capture service provision, with the feasibility study aiming to ascertain the required duration of time spent per pharmacy. It was found that one business day would be enough to reasonably sample the provision of unfunded services per pharmacy as no further significant information resulted from spending the extra business day per pharmacy with no new services being identified. Based on this finding it was decided to alter the study design from spending two days to spending one business day per pharmacy and increase the sample size of pharmacies recruited in the larger study.

Due to the nature of a pharmacy, typically patients do not spend a large amount of time waiting to access services. Patients accessing pharmacies tended to exit the pharmacy as soon as their query was addressed. These factors made it difficult to recruit patients for the onsite interviews. As a result, patients were approached at a higher frequency especially in the later stages of the study in order to ensure a larger number of patient interviewees. The sample size of patient interviews in this study was small but confirms the feasibility of conducting on-site interviews in the pharmacy.

Challenges also arose in regards to following up patients. Patients were telephoned three times and a voicemail or text message was sent leading to a follow up rate of $60 \%$. Despite this, no changes to the follow up method were made, as it was deemed sufficiently robust. After the feasibility study concluded it was decided that the concept of patients' willingness to pay would be further explored in the nationwide study. This is by way of an additional question at follow up, where the patient would be asked if they would still be willing to pay for the service given the outcome that resulted (if the patients' condition had resolved). This would give us data about ex-ante and expost willingness to pay and to investigate whether the patients' health outcomes influence their willingness to pay.

Interviews with pharmacy owners/managers did not yield any new information as compared to an earlier scoping study, about the methods utilised to achieve financial viability, other than cross subsidisation. This is in line with the pharmacist narratives captured in the preceding study. ${ }^{14}$ However, pharmacy owners and managers confirmed that funding is a barrier to service provision. As no new information was elucidated from these interviews, they were removed from the study design for the nationwide study.

\section{Limitations}

While the continuous observation time motion study provided many lessons for the design of the larger nationwide study, limitations also existed. Firstly, participation in this study was purely voluntary as no incentives were offered to any participants. This could have only captured pharmacies that were willing to share details of their day to day tasks as well as only those that provided unfunded services. Patient recruitment could have only captured patients who had strong views about pharmacy services with those who did not possess strong views choosing not to take part in the interviews.
The feasibility study did not compare the efficacies of different methodologies, rather only whether the continuous time motion study was appropriate to capture unfunded services.

Finally, the study was conducted during the summer months and may not have captured the seasonal variations in service provision.

\section{CONCLUSIONS}

The results of our study confirmed that a continuous observation time motion study was an appropriate method to utilise for the implementation of a nationwide investigation on unfunded pharmacy service provision. It is evident that the continuous observation time motion study allowed for the capture the provision of unfunded pharmacy services hence its appropriateness for the nationwide study. The study design also allowed for the identification of services which may be specific to certain pharmacies and those that are dictated by local governance in one location but not another.

Semi-structured patient interviews were an appropriate method to explore patient perceptions and valuations about accessing unfunded pharmacy services. Pharmacy owner/manager interviews did not highlight any previously unexplored method for achieving financial viability and therefore it was of little benefit to carry them out on a national level. Overall, the feasibility study confirmed that the methods proposed were appropriate to implement on a national level.

\section{ACKNOWLEDGEMENTS}

The authors would like to thank all the pharmacies, associated teams and patients that took part in the feasibility study.

\section{CONFLICT OF INTEREST}

The authors declare no conflicts of interest.

\section{FUNDING}

No funding was sought for this research.

\section{AUTHOR ROLES (CRediT)}

Conceptualization: SBD, CAM.

Data curation: YHAA.

Formal analysis: YHAA.

Funding acquisition: YHAA.

Investigation: YHAA.

Methodology: YHAA, SJH, SBD, CAM.

Project administration: CAM.

Resources: CAM.

Software: YHAA.

Supervision: SJH, SBD, CAM.

Validation: SJH.

Visualization: YHAA.

Writing - original draft: YHAA

Writing - review \& editing: YHAA, SJH, SBD, CAM. 


\section{References}

1. Ministry of Health New Zealand. Pharmacy action plan 2016 to 2020. https://www.health.govt.nz/system/files/documents/publications/pharmacy-action-plan-2016-to-2020.pdf (accessed Nov 5, 2017).

2. Canadian Pharmacists Association. Blueprint for pharmacy: transition in a new era. https://www.pharmacists.ca/cphaca/assets/File/pharmacy-in-canada/blueprint/Blueprint\%20Transition\%20Report 2015 ENG.pdf (accessed Oct 1, 2020).

3. Dunlop JA, Shaw JP. Community pharmacists' perspectives on pharmaceutical care implementation in New Zealand. Pharm World Sci. 2002;24(6):224-230. https://doi.org/10.1023/a:1021526425458

4. Smith AJ, Scahill SL, Harrison J, Carroll T, Medlicott NJ. Service provision in the wake of a new funding model for community pharmacy. BMC Health Serv Res. 2018;18(1):307. https://doi.org/10.1186/s12913-018-3120-z

5. Cruthirds DL, Hughes PJ, Weaver S. Value of pharmacy services to the healthcare system: an interdisciplinary assessment. Int J Pharm Pract. 2013;21(1):38-45. https://doi.org/10.1111/i.2042-7174.2012.00239.x

6. Scahill S, Harrison J, Carswell P, Shaw J. Health care policy and community pharmacy: implications for the New Zealand primary health care sector. N Z Med J. 2010;123(1317):41-51.

7. Kinsey H, Scahill S, Bye L, Harrison J. Funding for change: New Zealand pharmacists' views on, and experiences of, the community pharmacy services agreement. Int J Pharm Pract. 2016;24(6):379-389. https://doi.org/10.1111/ijpp.12266

8. Bernsten C, Andersson K, Gariepy Y, Simoens S. A comparative analysis of remuneration models for pharmaceutical professional services. Health Policy. 2010;95(1):1-9. https://doi.org/10.1016/j.healthpol.2009.11.008

9. Van Mil JWF, De Boer WO, Tromp TFJ. European barriers to the implementation of pharmaceutical care. Int J Pharm Pract. 2001;9(3):163-168

10. Cook M. DHB modelling sparks fears of funding cuts under new pharmacy contract. https://www.pharmacytoday.co.nz/article/news/dhb-modelling-sparks-fears-funding-cuts-under-new-pharmacy-contract (accessed May 9, 2018)

11. Chilton-Towle J. Pharmacists going extra mile fear future without funding https://www.pharmacytoday.co.nz/article/news/business/pharmacists-going-extra-mile-fear-future-without-funding (accesed Mar 23, 2018).

12. Zoio N. Pharmacists call for more funding for INR tests. https://www.pharmacytoday.co.nz/article/printarchive/pharmacists-call-more-funding-inr-tests (accessed Nov 10, 2017)

13. Torjesen I. Pharmacy funding cuts: the story so far. https://www.pharmaceutical-journal.com/news-andanalysis/features/pharmacy-funding-cuts-the-story-so-far/20202223.article?firstPass=false (accessed Mar 28, 2018).

14. Abdul Aziz YH, Heydon SJ, Duffull SB, Marra CA. What free services do pharmacists offer? Investigating the provision of unfunded pharmacy services in community pharmacies. Res Social Adm Pharm. 2021;17(3):588-594. https://doi.org/10.1016/j.sapharm.2020.05.007

15. Lopetegui M, Yen PY, Lai A, Jeffries J, Embi P, Payne P. Time motion studies in healthcare: what are we talking about?. J Biomed Inform. 2014;49:292-299. https://doi.org/10.1016/i.jbi.2014.02.017

16. Ampt A, Westbrook J, Creswick N, Mallock N. A comparison of self-reported and observational work sampling techniques for measuring time in nursing tasks. J Health Serv Res Policy. 2007;12(1):18-24. https://doi.org/10.1258/135581907779497576

17. Dreyer NA, Tunis SR, Berger M, Ollendorf D, Mattox P, Gliklich R. Why observational studies should be among the tools used in comparative effectiveness research. Health Aff (Millwood). 2010;29(10):1818-1825 https://doi.org/10.1377/hlthaff.2010.0666

18. Arain M, Campbell MJ, Cooper CL, Lancaster GA. What is a pilot or feasibility study? A review of current practice and editorial policy. BMC Med Res Methodol. 2010;10:67. https://doi.org/10.1186/1471-2288-10-67

19. Doody O, Doody CM. Conducting a pilot study: case study of a novice researcher. Br J Nurs. 2015;24(21):1074-1078 https://doi.org/10.12968/bjon.2015.24.21.1074

20. Bowen DJ, Kreuter M, Spring B, et al. How we design feasibility studies. Am J Prev Med. 2009;36(5):452-457. https://doi.org/10.1016/j.amepre.2009.02.002

21. Cavaye D, Lehnbom EC, Laba TL, El-Boustani E, Joshi R, Webster R. Considering pharmacy workflow in the context of Australian community pharmacy: A pilot time and motion study. Res Social Adm Pharm. 2018;14(12):1157-1162. https://doi.org/10.1016/i.sapharm.2018.01.003

22. Dean B, Barber N. Validity and reliability of observational methods for studying medication administration errors. Am J Health Syst Pharm. 2001;58(1):54-59. https://doi.org/10.1093/ajhp/58.1.54

23. Van de Mortel TF. Faking it: social desirability response bias in self-report research. Aust J Adv Nurs. 2008;25(4):40-48. 